\title{
BMJ Open Association of occasional smoking with total mortality in the population-based Tromsø study, 2001-2015
}

Maja-Lisa Løchen, ${ }^{1}$ Inger Torhild Gram, ${ }^{1}$ Jan Mannsverk, ${ }^{2}$ Ellisiv B Mathiesen, ${ }^{3,4}$ Inger Njølstad, ${ }^{1}$ Henrik Schirmer, ${ }^{3,5,6}$ Tom Wilsgaard, ${ }^{1}$ Bjarne K Jacobsen ${ }^{1}$

To cite: Løchen M-L, Gram IT, Mannsverk J, et al. Association of occasional smoking with total mortality in the population-based Troms $\emptyset$ study, 2001-2015. BMJ Open 2017;7:e019107. doi:10.1136/ bmjopen-2017-019107

- Prepublication history and additional material for this paper are available online. To view these files, please visit the journal online (http://dx.doi. org/10.1136/bmjopen-2017019107).

Received 10 August 2017 Revised 17 October 2017 Accepted 8 November 2017

\section{CrossMark}

${ }^{1}$ Department of Community Medicine, UiT The Arctic University of Norway, Troms $\emptyset$, Norway

${ }^{2}$ Division of Cardiothoracic and Respiratory Medicine, Department of Cardiology, University Hospital of North Norway, Troms $\emptyset$, Norway ${ }^{3}$ Department of Clinical Medicine, UiT The Arctic University of Norway, Tromsø, Norway

${ }^{4}$ Department of Neurology and Neurophysiology, The University Hospital of North Norway, Tromsø, Norway

${ }^{5}$ Department of Cardiology, Akershus University Hospital, Lørenskog, Norway

${ }^{6}$ Institute of Clinical Medicine, University of Oslo, Oslo, Norway

Correspondence to

Dr Maja-Lisa Løchen; maja-lisa.lochen@uit.no

\section{ABSTRACT}

Objectives There is a shift in the smoking population from daily smokers to light or occasional smokers. The knowledge about possible adverse health effects of this new smoking pattern is limited. We investigated smoking habits with focus on occasional smoking in relation to total mortality in a follow-up study of a Norwegian general population.

Setting A population study in Troms $\emptyset$, Norway.

Methods We collected smoking habits and relevant risk factors in 4020 women and 3033 men aged 30-89 years in the Troms $\emptyset$ Study in 2001. The subjects were followed up regarding total mortality through June 2015.

Results Among the participants, there were 7\% occasional smokers. Occasional smokers were younger, more educated and used alcohol more frequently than other participants. A total of 766 women and 882 men died during the follow-up. After the adjustment for confounders, we found that occasional smoking significantly increased mortality by $38 \%$ (95\% Cl $8 \%$ to $76 \%$ ) compared with never smokers. We report a dose-response relationship in the hazards of smoking (daily, occasional, former and never smoking).

Conclusions Occasional smoking is not a safe smoking alternative. There is a need for information to the general public and health workers about the health hazards of occasional smoking. More work should be done to motivate this often well-educated group to quit smoking completely.

\section{INTRODUCTION}

Smoking is an important preventable risk factor for disease and premature death. There is a shift in the smoking population from daily, addicted tobacco users to light or occasional smokers without similar nicotine dependence. Smoking prevalence in Norway has been nearly halved during the last decade, $12 \%$ of Norwegian men and women aged 16-74 years were daily smokers in $2016 .{ }^{1}$ While daily smoking is declining, the prevalence of intermittent or occasional smoking in Norway has remained quite stable during the last decade, with $9 \%$ occasional smokers in 2016 compared with $11 \%$ in 2005 . Occasional smoking is frequently found among young,

\section{Strengths and limitations of this study}

- A longitudinal study including 7053 men and women who participated in a screening and gave information about smoking habits in 2001 ( $65 \%$ and $70 \%$ of the invited men and women, respectively) with complete mortality follow-up through June 2015.

- Information of a number of possible confounding variables was available.

- Information about smoking history was collected from several questions in two questionnaires at baseline and was self-reported with no objective measures of tobacco exposure.

- Occasional smokers is an unstable and heterogeneous group of former daily smokers trying to quit, persistent occasional smokers and former quitters who are occasional smokers for a period, and this instability is mirrored by somewhat inconsistent answers to the smoking history questions.

- No information is available regarding changes in smoking habits during the follow-up.

educated people and women. ${ }^{2}$ In 2013 , the Norwegian Directorate of Health launched a campaign to reach these segments of occasional smokers, focusing particularly on the relationship between occasional smoking and acute myocardial infarction. ${ }^{3}$

The literature so far is not large. A cohort study from Finland indicated that occasional smoking carried almost similar effect on death from any cause as daily smoking in men. ${ }^{4}$ A review from 2010 emphasised the need for more cohort studies explicitly comparing risk in daily smokers, occasional smokers and non-smokers. ${ }^{1}$ In 2016, a large cohort study from the USA among low-intensity smokers over lifetime, found that they had higher mortality risk than never smokers. ${ }^{5}$ More information about the adverse health effects of this new smoking pattern is needed both for the general population and for health professionals. In this population-based prospective study from Norway, we aimed to analyse smoking habits with a focus on 
occasional smoking in relation to total death risk in a 14-year follow-up.

\section{METHODS}

\section{Tromsø Study}

The Tromsø Study is a population-based prospective multipurpose study. ${ }^{6}$ Seven surveys have been conducted, the first in 1974 and the last in 2015-2016. To the fifth survey (Tromsø 5), conducted in 2001, 10353 persons were invited. These individuals were men and women who lived in the municipality of Troms $\varnothing$ and had participated in the second visit of the fourth survey in 19941995 (the majority were 62-81 years old in 2001) as well as all women and men aged 30, 40, 45, 60 or 75 years. A total of 3511 men and 4619 women aged $30-89$ years attended; attendance rates of $75.7 \%$ and $80.8 \%$, respectively. The participants received a questionnaire along with the invitation and were asked to bring the completed form when they came to the physical examination including also non-fasting blood samples. The questionnaire included questions concerning, among other topics, smoking habits. People who attended the physical examination received a second questionnaire, which they were asked to complete and return in by mail. This questionnaire included a question on occasional smoking. The study design and data collection are described in some more detail elsewhere. ${ }^{6}$ An English translation of the questionnaires is available at the Tromsø Study website. ${ }^{7}$

The study adhered to the tenets of the Declaration of Helsinki and the Regional Committee for Medical and Health Research Ethics and the Norwegian Data Protection authority approved the study. All participants gave written informed consent.

\section{Questionnaires and measurements}

We based information about history of smoking on several questions in two questionnaires. A total of 7999 men and women answered the question concerning daily smoking on the first questionnaire ('Do you/did you smoke daily?' with the answer alternatives 'Yes, now', 'Yes, previously' or 'Never'). In addition, there were questions about duration of smoking and the number of cigarettes smoked on a daily basis (if ever smoker). More than $90 \%$ of ever smokers gave information concerning cigarettes per day and the duration of smoking (years), making computation of the number of pack-years possible.

Furthermore, 7116 subjects answered the question concerning smoking on the next questionnaire ('Do you smoke?' with the answer alternatives 'Yes, daily', 'Yes, sometimes' and 'No, never'), thereby giving information about occasional, but not daily smoking. There were, however, some inconsistencies, like subjects who reported to be a current daily smoker on the second questionnaire, but a previous or never smoker on the former or a never smoker on the second questionnaire and current smoker on the first. These subjects were excluded from the analyses. We therefore identified four groups of subjects, including a total of 4020 women and 3033 men (68\% of the invited population).

1. Consistent daily smokers.

2. Occasional smokers: subjects who stated to be an occasional smoker in the second questionnaire and gave information about smoking habits in the first questionnaire.

3. Former smokers: subjects who stated to be a never smoker in the second questionnaire, but stated to be a former smoker in the first questionnaire, essentially identifying a group of subjects who had been smokers, but never smoked now, not even occasionally. These subjects may be at risk because of previous smoking.

4. Never smokers: consistent never smokers.

The questionnaires also included a question regarding passive smoking ('Do you currently, or did you previously live together with a daily smoker after your 20th birthday?' with two answer alternatives, 'Yes' and 'No'). Pack-years was computed as (number of cigarettes per day $\times$ duration of smoking)/20. An English translation of the questionnaires is available at the Troms $\varnothing$ Study website ${ }^{7}$ and as online supplementary files 1 and 2 .

Frequency of use of alcohol last year was assessed by the question 'approximately how often have you during the last year consumed alcohol?' with eight answer alternatives from 'never consumed alcohol' to '4-7 times a week'. The highest attained level of education was self-reported and classified as follows: (1) primary/partly secondary education (up to 9 years of schooling); (2) upper secondary education (10-12 years of schooling); (3) tertiary education, short (college/university $<4$ years) and (4) tertiary education, long (college/university $\geq 4$ years).

Height and weight measurements were performed in light clothing and without footwear. Waist circumference was measured without outerwear by using a measuring tape across the belly button. The participant was taken into a separate room with only a nurse present to measure the blood pressure. The blood pressure was measured three times at $1 \mathrm{~min}$ intervals on one arm after the participant had been seated for 2 min using an automatic device (Dinamap Vital Signs Monitor 1846; Criticon, Tampa, Florida, USA). The mean of the two last measurements was used in the present analyses.

The blood test included the measurement of serum total cholesterol, serum high-density lipoprotein (HDL) cholesterol and triglycerides. Analysis of serum total cholesterol and triglycerides was performed by enzymatic colorimetric methods with commercial kits (CHOD-PAP; Boehringer-Manheim, Mannheim, Germany). HDL cholesterol was measured after precipitation of lower density lipoproteins with manganese chloride. All the analyses were performed by the Department of Clinical Chemistry, University Hospital of North Norway in Tromsø.

\section{Follow-up and statistical analyses}

The National Causes of Death Registry covers individuals registered as residents of Norway at the time of their death, 
without regard to whether the death took place in Norway or abroad. The subjects were followed up regarding total mortality from the day they attended the Troms $\varnothing 5$ survey and to the date of death, emigration from Norway or 30 June 2015, whichever came first. There were 1648 deaths during follow-up. The mean (minimum and maximum) follow-up was 12.5 (0.2-14.3) years.

Baseline characteristics were presented as mean (SD) or percentages (number of subjects). The simple descriptive statistical analyses included analyses of variance and $\mathrm{X}^{2}$ tests and the $\mathrm{P}$ values were $\mathrm{P}$ for homogeneity. The relationships between smoking habits and mortality were assessed by Cox proportional hazard regression analyses with attained age as the continuous time variable, including 95\% CIs for the HRs. Consistent never smokers constituted the reference category. Adjustments were performed for other significant predictors for mortality in this cohort after adjustment for sex and attained age, that is, education, body mass index (BMI), total serum cholesterol and serum triglycerides.

\section{RESULTS}

Mean age for both sexes (4020 women and 3033 men) was about 60 years. Among the participants, there were $33 \%$ never smokers, $34 \%$ former smokers, $7 \%$ occasional smokers and $26 \%$ consistent daily smokers (table 1 ). In both sexes, occasional smokers tended to be younger than other smokers. A higher proportion of occasional smokers had high education levels and weekly use of alcohol. Passive smoking was most common in current smokers. The use of snuff (both current and previous use) was most prevalent in men and in occasional smokers. Occasional and daily smokers had lower BMI compared with never and former smokers.

\begin{tabular}{|c|c|c|c|c|c|}
\hline & Never smokers & $\begin{array}{l}\text { Former } \\
\text { smokers }\end{array}$ & $\begin{array}{l}\text { Occasional } \\
\text { smokers }\end{array}$ & $\begin{array}{l}\text { Consistent } \\
\text { daily smokers }\end{array}$ & $P$ value* \\
\hline Women, n (\%) & $1648(41.0)$ & $1061(26.4)$ & $257(6.4)$ & $1054(26.4)$ & \\
\hline No of pack-years & 0 & $9.2(9.1)$ & $6.1(7.4)$ & $17.4(11.0)$ & $<0.001 \dagger$ \\
\hline Age (years) & $61.8(13.5)$ & $60.2(13.3)$ & $53.3(15.3)$ & $56.1(13.4)$ & $<0.001$ \\
\hline Education >12 years (\%) & $29.4(461)$ & $26.9(277)$ & $39.4(98)$ & $23.1(235)$ & $<0.001$ \\
\hline$\%$ exposed for passive smoking & $58.3(945)$ & $77.8(807)$ & 75.2 (191) & $86.1(880)$ & $<0.001$ \\
\hline$\%$ with weekly alcohol use & $20.9(330)$ & $28.3(291)$ & $31.7(79)$ & $27.8(288)$ & $<0.001$ \\
\hline Ever use of snuff & $0.4(5)$ & $1.3(10)$ & $2.1(5)$ & $0.6(6)$ & 0.02 \\
\hline $\mathrm{BMI}\left(\mathrm{kg} / \mathrm{m}^{2}\right)$ & $26.9(4.6)$ & $27.4(4.6)$ & $25.5(4.4)$ & $24.9(4.2)$ & $<0.001$ \\
\hline Waist circumference $(\mathrm{cm})$ & $85.0(11.7)$ & $86.7(12.3)$ & $82.1(11.1)$ & $81.3(11.0)$ & $<0.001$ \\
\hline Systolic blood pressure $(\mathrm{mm} \mathrm{Hg})$ & $141.4(23.4)$ & $138.1(22.8)$ & $130.4(23.1)$ & $132.7(22.6)$ & $<0.001$ \\
\hline Total cholesterol (mmol/L) & $6.3(1.2)$ & $6.3(1.2)$ & $6.0(1.3)$ & $6.3(1.2)$ & 0.003 \\
\hline HDL cholesterol (mmol/L) & $1.6(0.4)$ & $1.6(0.4)$ & $1.6(0.4)$ & $1.5(0.4)$ & $<0.001$ \\
\hline Triglycerides (mmol/L) & $1.4(0.8)$ & $1.4(0.7)$ & $1.3(0.7)$ & $1.5(0.9)$ & 0.006 \\
\hline Men, n (\%) & $698(23.1)$ & $1302(42.9)$ & $252(8.3)$ & $781(25.8)$ & \\
\hline No of pack-years & 0 & $17.5(16.0)$ & $10.8(13.3)$ & $22.6(13.2)$ & $<0.001 \dagger$ \\
\hline Age (years) & $55.9(14.6)$ & $65.0(11.5)$ & $55.5(14.6)$ & $58.2(13.4)$ & $<0.001$ \\
\hline Education >12 years (\%) & $40.9(278)$ & $22.3(280)$ & $41.9(104)$ & $23.4(176)$ & $<0.001$ \\
\hline$\%$ exposed for passive smoking & $35.8(247)$ & $76.1(969)$ & $69.0(171)$ & $84.4(637)$ & $<0.001$ \\
\hline$\%$ with weekly alcohol use & $34.8(240)$ & $35.8(458)$ & $47.6(118)$ & $40.0(309)$ & $<0.001$ \\
\hline Ever use of snuff & $4.9(31)$ & $5.5(64)$ & $11.7(29)$ & $5.2(40)$ & $<0.001$ \\
\hline BMI $\left(\mathrm{kg} / \mathrm{m}^{2}\right)$ & $26.8(3.5)$ & $27.2(3.6)$ & $26.7(3.6)$ & $26.0(3.5)$ & $<0.001$ \\
\hline Waist circumference $(\mathrm{cm})$ & $94.1(9.4)$ & $97.0(10.6)$ & $94.5(10.5)$ & $93.6(10.2)$ & $<0.001$ \\
\hline Systolic blood pressure $(\mathrm{mm} \mathrm{Hg})$ & $138.7(20.1)$ & $143.7(20.6)$ & $135.2(16.5)$ & $137.1(19.1)$ & $<0.001$ \\
\hline Total cholesterol (mmol/L) & $6.0(1.1)$ & $6.0(1.1)$ & $6.0(1.1)$ & $6.1(1.1)$ & 0.36 \\
\hline HDL cholesterol (mmol/L) & $1.3(0.3)$ & $1.4(0.4)$ & $1.3(0.4)$ & $1.3(0.3)$ & 0.006 \\
\hline Triglycerides (mmol/L) & $1.7(1.0)$ & $1.7(1.0)$ & $1.6(0.8)$ & $1.7(1.1)$ & 0.56 \\
\hline
\end{tabular}

Values are mean (SD) or percentages (number of subjects). There were some missing values for some variables.

${ }^{*} \mathrm{P}$ value for homogeneity.

†P value not including never smokers.

BMI, body mass index; HDL, high-density lipoprotein. 
Table 2 Relationships between smoking habits and total mortality. A 14-years follow-up. The Tromsø study

\begin{tabular}{lcccccl}
\hline & Subjects, $\mathbf{n}(\%)$ & Deaths, $\mathbf{n}(\%)$ & $\mathbf{H R}^{*}$ & $\mathbf{9 5 \%} \mathbf{C l}$ & $\mathbf{H R} \boldsymbol{9}$ & $\mathbf{9 5 \%} \mathbf{C l}$ \\
\hline Consistent daily smokers & $1835(26.0)$ & $468(28.4)$ & 2.13 & 1.86 to 2.43 & 2.05 & 1.78 to 2.37 \\
Occasional smokers & $509(7.2)$ & $88(5.3)$ & 1.32 & 1.05 to 1.66 & 1.38 & 1.08 to 1.76 \\
Former smokers & $2363(33.5)$ & $633(38.4)$ & 1.14 & 1.00 to 1.30 & 1.18 & 1.03 to 1.35 \\
Never smokers & $2346(33.3)$ & $459(27.9)$ & 1.00 & Reference & 1.00 & Reference \\
Total & $7053(100)$ & $1648(100)$ & & & & \\
\hline
\end{tabular}

${ }^{*}$ Adjusted for age and gender.

†Adjusted for age, gender, education, BMI, serum cholesterol and serum triglycerides.

BMI, body mass index.

Table 2 confirms that both consistent daily smoking and occasional smoking were associated with increased all-cause mortality. A total of 766 women and 882 men died during the follow-up. Although the age-adjusted HR associated with occasional smoking compared with never smokers was somewhat higher in women $(\mathrm{HR}=1.59(95 \%$ CI 1.15 to 2.20$)$ ) than in men ( $\mathrm{HR}=1.23$ (95\% CI 0.88 to 1.73)), the overall relationships between smoking habits and total mortality were not statistically significantly different ( $\mathrm{P}$ value for interaction=0.07) and the $\mathrm{P}$ value for the difference in the relationships with occasional smoking was higher $(\mathrm{P}=0.3)$ (data not shown). Due to the relatively low number of deaths among the individuals who smoked occasionally, we merged results for men and women and adjusted for gender. Adjustments for other significant predictors for mortality in this cohort (education, BMI, total serum cholesterol and serum triglycerides) changed the point estimates only marginally and the conclusions were unaffected (table 2). This was also the case when further adjustments for the frequency of use of alcohol and passive smoking were undertaken.

In a separate set of analyses, we included only men and women who at baseline indicated to be free of ischaemic heart disease (reporting no myocardial infarction or angina pectoris). In this group of 6121 subjects, there were 1232 deaths. This exclusion of individuals had minimal impact on the point estimate for the relationship between occasional smoking and total mortality; $\mathrm{HR}=1.27$ (95\% CI 0.97 to 1.67$)$ compared with $\mathrm{HR}=1.32$ (95\% CI 1.05 to 1.66$)$ in the analyses including the total population (table 2).

Detailed information about smoking history (the number of pack-years) was missing for 543 ever smokers. In separate analyses, we restricted the analytical cohort to 4164 ever smokers (current daily smokers, occasional smokers and previous smokers) with complete information about the number of pack-years. There were 1013 deaths. The age-adjusted and gender-adjusted mortality in occasional smokers were not higher than in former smokers (HR=1.12, 95\% CI 0.87 to 1.43 ), and adjusting for the number of pack-years in addition to age and gender confirmed this. If anything, the relationship with occasional smoking was somewhat stronger, but still not statistically significant.
In a separate set of analyses, we restricted the analyses to subjects aged 79 years or below at follow-up, thus disregarding information from follow-up after the age of 80 years. A total of 6886 men and women and 754 deaths were included in the analyses. The results with regard to mortality in occasional smokers were essentially unchanged. Similarly as for the analyses including all subjects, we also in this situation restricted the analytical cohort to 4094 ever smokers with 526 deaths and adjusted for the number of pack-years. The results were as for all subjects, also including follow-up after the age of 79 years.

\section{DISCUSSION}

The present prospective cohort study shows that occasional smoking significantly increased mortality by more than $30 \%$ compared with never smokers. Results were not substantially changed when analyses were restricted to those aged below 80 years at the follow-up. We found that the $7 \%$ occasional smokers constituted the youngest group of individuals, they used alcohol more frequently and they had higher educational level compared with the other study attendees.

Our results are in line with findings from other surveys of occasional smokers; they are younger than daily smokers and their level of education is more similar to non-smokers. ${ }^{38}$ A Finnish prospective cohort study studied occasional smoking habits at baseline as risk factor for total mortality. ${ }^{4}$ Their finding was about similar to ours for men, while female occasional smokers did not have an increased mortality risk. However, there was no significant difference in the association between occasional smoking and total mortality between the sexes, similar to our findings. A recent large cohort study from the USA included self-reports of lifetime smoking history and showed that persons who smoked $<1$ or $1-10$ cigarettes per day over their lifetime had higher all-cause mortality risks than never smokers. ${ }^{5}$ A previous large population-based Norwegian cohort study found that even very light smoking (one to four cigarettes per day) was associated with a significantly $50 \%-60 \%$ increased all-cause mortality, ${ }^{9}$ while a British study demonstrated a significant HR of 1.21 for light smoking compared with never smoking. ${ }^{10}$ A study from the USA experienced 
a more than two times higher mortality in very light smoking females. ${ }^{11}$ Light smoking may be comparable to occasional smoking when it comes to risk of all-cause mortality in our study. Differences in risk compared with the present study may be due to different study populations and length of follow-up as well as various abilities to control for confounders. We have previously shown that light smoking as well as passive smoking carried higher hazards for myocardial infarction in women. ${ }^{12}$ Recently, a British cohort study with long follow-up found that light smoking at baseline carried a higher mortality risk in women than in men. ${ }^{10}$ Our results give some risk estimates supporting this finding, but we did not find a statistically significant interaction and therefore merged the data for the two genders.

Norway has a strong record on national tobacco control policies since the 1970s. The trend of occasional smoking might be influenced by the ongoing marginalisation of smoking and increasing restrictions. Studies have shown that a large proportion of occasional smokers do not regard themselves as smokers. ${ }^{8}$ There is a common belief, based in part on successful tobacco industry marketing to so-called 'health-conscious smoking', that occasional smoking is safer than daily smoking. ${ }^{13}$ A Norwegian Directorate of Health survey in 2013, conducted before a campaign to reach occasional smokers, confirmed this. One-third of the occasional smokers did not believe their smoking would cause any harm to their health. ${ }^{3}$ We do not have data to confirm these conceptions, but the relatively high education level of occasional smokers in the present study suggests that they are well aware of the hazards of daily smoking, as well educated people are, but may consider occasional smoking far less detrimental or maybe without any health risks. Moreover, it is shown that occasional smokers are not free of nicotine dependence and that their smoking appears to be driven to some degree by the same cigarette craving that affects daily smokers, explaining why many occasional smokers have difficulty quitting. ${ }^{14}$ This is important knowledge for health professionals working with smoking cessation in occasional smokers.

In 2006-2010, approximately $10 \%$ of Norwegians aged 16-74 years were occasional smokers. ${ }^{1}$ Our slightly lower prevalence may be because no subjects in our study were below 30 years, and occasional smoking is known to be more frequent among younger individuals. The use of snuff (snus) has been increasingly popular in Norway, particularly among adolescents and young adults. ${ }^{1}$ Approximately $3 \%$ of the subjects included in our study reported ever use of snuff, and it was not associated with increased mortality, but with occasional smoking (table 1).

Population studies have risk of selection bias because those who accept the invitation to participate in the study may not be representative of the whole target population. Non-response is often linked to exposure status, which implies that, for example, individuals with health issues, smokers and others with unhealthy lifestyle may be less likely to attend the survey compared with non-smokers. We have previously reported lower mortality in participants to the Troms $\varnothing$ Study according to attendance. ${ }^{6}$ This bias would influence our findings only if the association between smoking habits and total mortality is different in the $68 \%$ of the invited population who were included in the analyses than in the remaining $32 \%$.

The participants in the Troms $\varnothing$ Study reported smoking habits on a self-administered questionnaire that may imply information bias. We have no objective measures of tobacco exposure like cotinine or thiocyanate. A previous Norwegian study showed that the relation between self-reported smoking habits and the measure of serum thiocyanate was strong if the question was asked in a neutral setting. ${ }^{15}$ As the questions about smoking were asked in a neutral setting, we believe that the validity was good, although we recognise that the smoking habits probably are under-reported.

It is a limitation that we have not been able to include information regarding changes in smoking habits during the follow-up. The smoking prevalence in our community has declined. ${ }^{16}$ In Tromsø 5 (2001), it was $28 \%$ current smokers, 20\% in Tromsø 6 (2007-2008) and 14\% in Troms $\varnothing 7$ (2015-2016). Seven per cent of the Troms $\varnothing 7$ population reported to be occasional smokers, $19 \%$ had previously been. ${ }^{7}$

A particular problem in studies of the health risks related to occasional smoking is that occasional smoking is a rather unstable category consisting of a heterogeneous group of former daily smokers trying to quit, persistent occasional smokers who might regard their risk as little and former quitters who have resumed as occasional smokers for a period. This instability is mirrored by the inconsistent answers to the smoking history questions we used for categorisation in our study. Among the subjects who stated to be occasional smokers on the second questionnaire in our study population, $28 \%, 60 \%$ and $13 \%$, respectively, reported to be current, ex-daily smokers and never daily smokers on the first questionnaire.

A total of 3729 of the subjects included in our analyses also answered a question concerning occasional smoking in the Troms $\varnothing 6$ survey in 2007-2008, and information about occasional smoking was therefore available from both surveys. We found that $39 \%$ of those who at baseline (in 2001) were classified as occasional smokers reported the same in 2007-2008 (Tromsø 6). When comparing with their self-reported classification as current, ex-smokers and never smokers in 2007-2008, $13 \%$ of occasional smokers in 2001 reported to be current daily smokers in 2007-2008 and 65\% reported to be previous daily smokers. Thus, the changing smoking habits among the occasional smokers make it difficult to precisely assess the strength of the relationship between occasional smoking and total mortality. Only registration of long-term smoking habits can answer this question.

Another limitation in our study is that we lack information about usage patterns among the occasional smokers, 
for example, how often they smoked and how many cigarettes they smoked per occasion.

Our study had the strength of a large cohort with a prospective design, high participation rate and a quite long and complete follow-up. Moreover, we were able to adjust for baseline levels of other significant risk factors for total mortality in this population. The results are probably valid for other European populations, but similar cohort studies should be conducted in other populations in order to determine the exact adverse effects of occasional smoking. In particular, there is a need for larger studies as there were relatively few occasional smokers in our study and therefore only 88 deaths.

In conclusion, in line with a few others, this study demonstrates that occasional smoking is not a safe alternative; it increases mortality. We report a dose-response relationship in the hazards of smoking (daily, occasional, former and never smoking). Governmental and non-governmental tobacco control policy-makers should intensify the information about the health hazards of occasional smoking as well as work towards increased restrictions. Occasional smokers make up about one-third of all current smokers in the Norwegian population, and more work should be done to motivate this usually well-educated group to quit smoking completely.

Contributors M-LL and BKJ: conceived and designed the research. BKJ: performed the analyses. M-LL and BKJ: drafted the manuscript. All the authors made critical revision of the manuscript for key intellectual content. All the authors have read and approved the submitted version of the manuscript.

Funding The publication charges for this article have been funded by a grant from the publication fund of UiT The Arctic University of Norway.

Competing interests None declared.

Ethics approval The Troms $\emptyset$ Study was approved by the Data Inspectorate of Norway and the Regional Committee of Medical and Health Research Ethics, North Norway.

Provenance and peer review Not commissioned; externally peer reviewed.

Data sharing statement № additional data available.

Open Access This is an Open Access article distributed in accordance with the Creative Commons Attribution Non Commercial (CC BY-NC 4.0) license, which permits others to distribute, remix, adapt, build upon this work non-commercially, and license their derivative works on different terms, provided the original work is properly cited and the use is non-commercial. See: http://creativecommons.org/ licenses/by-nc/4.0/

(C) Article author(s) (or their employer(s) unless otherwise stated in the text of the article) 2017. All rights reserved. No commercial use is permitted unless otherwise expressly granted.

\section{REFERENCES}

1. Statistics Norway. Smoking habits. 2017 https://www.ssb.no/en/ helse/statistikker/royk

2. Schane RE, Ling PM, Glantz SA. Health effects of light and intermittent smoking: a review. Circulation 2010;121:1518-22.

3. Nylenna A. Occasional smoking: a new campaign target in Norway. Lancet 2013;381:708-9.

4. Luoto R, Uutela A, Puska P. Occasional smoking increases total and cardiovascular mortality among men. Nicotine Tob Res 2000;2:133-9.

5. Inoue-Choi M, Liao LM, Reyes-Guzman C, et al. Association of long-term, low-intensity smoking with all-cause and cause-specific mortality in the national institutes of health-AARP diet and health study. JAMA Intern Med 2017;177:87-95.

6. Jacobsen BK, Eggen AE, Mathiesen EB, et al. Cohort profile: the Troms $\varnothing$ study. Int J Epidemiol 2012;41:961-7.

7. UiT The Arctic University of Norway. The Troms $\varnothing$ study. 2017. http:// www.tromsostudy.com

8. Schane RE, Glantz SA, Ling PM. Nondaily and social smoking: an increasingly prevalent pattern. Arch Intern Med 2009;169:1742-4.

9. Bjartveit K, Tverdal A. Health consequences of smoking 1-4 cigarettes per day. Tob Control 2005;14:315-20.

10. Hurley MA. Light smoking at base-line predicts a higher mortality risk to women than to men; evidence from a cohort with long follow-up. BMC Public Health 2014;14:95.

11. Holahan CK, Holahan CJ, North RJ, et al. Smoking status, physical health-related quality of life, and mortality in middle-aged and older women. Nicotine Tob Res 2013;15:662-9.

12. Iversen B, Jacobsen BK, Løchen ML. Active and passive smoking and the risk of myocardial infarction in 24,968 men and women during 11 year of follow-up: the Tromsø study. Eur J Epidemiol 2013;28:659-67.

13. Schane RE, Glantz SA, Ling PM. Social smoking implications for public health, clinical practice, and intervention research. Am J Prev Med 2009;37:124-31.

14. Shiffman S, Dunbar MS, Kirchner TR, et al. Cue reactivity in non-daily smokers: effects on craving and on smoking behavior. Psychopharmacology 2013;226:321-33.

15. Foss OP, Haug K, Hesla PE, et al. Can we rely on self-reported smoking habits?. Tidsskr Nor Laegeforen 1998;118:2165-8.

16. Eggen AE, Mathiesen EB, Wilsgaard T, et al. Trends in cardiovascular risk factors across levels of education in a general population: is the educational gap increasing? The Troms $\varnothing$ study 1994-2008. J Epidemiol Community Health 2014;68:712-9. 